\title{
Sildenafil improved erectile dysfunction and quality of life in men with comorbid mild to moderate depression
}

Seidman SN, Roose SP, Menza MA, et al. Treatment of erectile dysfunction in men with depressive symptoms: results of a placebo-controlled trial with sildenafil citrate. Am J Psychiatry 2001 Oct;158:1623-30.

\section{QUESTION: In men with erectile dysfunction (ED) and mild-to-moderate depression, does depression affect ED treatment response to sildenafil and does improvement in ED affect depression?}

\section{Design}

Randomised \{allocation concealed*\}†, blinded \{patients, clinicians, data collectors, and outcome assessors $\} \dagger^{* *}$ placebo controlled trial with 12 weeks of follow up.

\section{Setting}

20 urology clinics in the USA.

\section{Patients}

152 men (mean age 56 y) who had ED for $\geqslant 6$ months (mean duration 6 y); a stable relationship with a female partner for $\geqslant 6$ months; a Diagnostic and Statistical Manual of Mental Disorders, 4th ed, diagnosis of depressive disorder not otherwise specified; and a Hamilton Depression Rating Scale score of $\geqslant 12$. Exclusion criteria included presence of another axis I psychiatric disorder, current use of nitrates or any antidepressant medication, and abnormal serum hormone concentrations. $89 \%$ of patients were included in the analysis.

\section{Intervention}

74 men were allocated to flexible dose (25 to $100 \mathrm{mg}$ ) sildenafil begun at $50 \mathrm{mg}$, and 78 were allocated to matching placebo, both to be taken 1 hour before sexual activity.

\section{Main outcome measures}

Changes in sexual function, depressive symptoms, quality of life, and adverse effects.

\section{Main results}

Analysis was by intention to treat. More patients in the sildenafil group were classified as treatment responders (improvement in erections and ability to have sexual intercourse and a score of $\geqslant 21$ on the erection function domain of the International Index of Erectile Function scale) than were patients in the placebo group (table). Improvement in ED was highly correlated with improvement in depressive symptoms and quality of life, regardless of treatment type ( $76 \%$ of ED responders $v 14 \%$ of ED non-responders achieved depression remission). Treatment related adverse effects occurred in more patients who received sildenafil than placebo $(47 \% v$ $13 \%,\{\mathrm{p}<0.001\} \ddagger)$.

\section{Conclusions}

In men with erectile dysfunction and mild to moderate depression, sildenafil was associated with improved erectile dysfunction. Improvement in erectile dysfunction was associated with improvement in depressive symptoms and quality of life.

*See glossary.

$\dagger$ Information provided by author.

$\ddagger$ p Value calculated from data in article.
Source of funding:

Pfizer Inc.

For correspondence: Dr S N Seidman, Columbia University, New York, USA. sns5@columbia.edu.

Sildenafil v placebo for erectile dysfunction and mild to moderate depressive illness at 12 weeks

\begin{tabular}{lllll} 
Outcome & Sildenafil & Placebo & RBI $(95 \%$ Cl) & NNT (CI) \\
Treatment response & $73 \%$ & $14 \%$ & $409 \%(191$ to 830$)$ & 2 (2 to 3) \\
\hline
\end{tabular}

§Abbreviations defined in glossary; RBI, NNT, and $\mathrm{Cl}$ calculated from data in article.

IImprovement in erections and ability to have sexual intercourse and a score of $\geqslant 21$ on the erection function domain of the International Index of Erectile Function scale.

\section{COMMENTARY}

The study by Seidman et al does not advocate sildenafil as treatment for primary mild to moderate depression. Rather, it adds to the clinical context and options available when approaching the common comorbidity of ED and mild to moderate depression. Although the study sets out to show that sildenafil can effectively treat ED in men with mild to moderate depression, it also sheds some light on the question of "Which is first, mild to moderate depression or ED?" By showing that alleviation of ED results in improved mild to moderate depression and better quality of life, the study generates more questions: Which should be treated first, mild to moderate depression or ED? Should they be treated concomitantly? What happens after 12 weeks? Do these findings apply to patients with major depressive disorder?

This study has some limitations. It was only 12 weeks in duration. Although the completion rates were relatively high ( $88 \%$ [sildenafil] and $77 \%$ [placebo]), the drop outs were not adequately accounted for, and out of the 152 patients who received $\geqslant 1$ dose of study medication, 16 were not assessed after randomisation.

Use of sildenafil comes at a price. Aside from the cost of the medication, the difference in rate of side effects was statistically significant (47\% [sildenafil] $v 13 \%$ [placebo]). The most common side effects (flushing, headache, dyspepsia, coloured tinges, and increased perception of brightness of lights) were generally mild to moderate and transient; the higher completion rate for sildenafil implies tolerability. No deaths were reported.

A growing number of symptom clusters (eg, social phobia, post-traumatic stress disorder, and generalised anxiety disorder) is being treated with antidepressant pharmacotherapy. This study reminds us that not all symptom clusters are primary and that there is need and value in evaluating for root issues. Maintaining "secondary" depression in assessment differentials, then considering and treating causes (ED, lack of sleep or exercise, alcohol, drugs [prescribed or otherwise], hormones, or domestic violence), can be therapeutic.

In men similar to those studied, one can safely consider closely followed primary treatment of ED with sildenafil.

Stephen A Wilson, MD UPMC St. Margaret Family Practice Residency Pittsburgh, Pennsylvania, USA 